\title{
Use of Telehealth Amid the COVID-19 Pandemic: Experiences of Mental Health Providers Serving Rural Youth and Elderly in Pennsylvania
}

\author{
Juliana Svistova $^{1} \cdot$ Christopher Harris $^{1} \cdot$ Barbe Fogarty $^{1} \cdot$ Christina Kulp $^{1} \cdot$ Ahyoung Lee $^{2}$ (D)
}

Accepted: 23 November 2021 / Published online: 30 November 2021

(c) The Author(s), under exclusive licence to Springer Science+Business Media, LLC, part of Springer Nature 2021

\begin{abstract}
Stay-at-home orders and public health safety concerns precipitated by the COVID-19 pandemic facilitated rapid changes in policy and reimbursement regulations and resulted in the sudden uptake of telehealth in mental health practices across the United States. This study explored how mental health service providers experienced the use of telehealth in serving their rural clients who are youth and older adults. A purposive sampling strategy was employed to identify and recruit mental health service providers and insurance agency representatives for this study. By means of online focus groups, this statewide study explored the experiences of 147 mental health and public insurance providers using telehealth to serve rural youth and elderly in Pennsylvania amid the pandemic in 2020. NVivo 12 qualitative analysis software was used in data analysis. The findings suggest that telehealth is perceived as both: silver linings during the pandemic (service continuation during the pandemic, improved parental involvement and responsiveness, easing the transportation challenges, and decrease in no-show rates) and some roadblocks to success (Not for every youth!, Technology challenges among the older adults, and "Dead zones" without internet and cellphone reception). Policy and practice recommendations are suggested including incentives for proactive telehealth uptake, telehealth parity laws and reimbursement policies, and incentivizing innovative use of technology for specific populations and therapeutic modalities. Continuous policy support and organizational efforts to provide customized telemental health are called for to remediate rural disparities in access to mental health services beyond the pandemic period.
\end{abstract}

Keywords Telehealth $\cdot$ Mental health services $\cdot$ Rural disparity $\cdot$ Youth $\cdot$ Elderly $\cdot$ COVID-19

\section{Introduction}

Stay-at-home orders and safety concerns precipitated by the COVID-19 pandemic facilitated rapid changes in policy and reimbursement regulations (The Centers for Medicare \& Medicaid Services, 2020) and resulted in sudden uptake and implementation of telehealth in medical and mental health practices in both urban and rural areas. Following Pennsylvania Governor Wolf's "Proclamation of Disaster" related to the covid-19 pandemic (Wolf, 2020), the Department of Human Services Office of Mental Health and Substance

Ahyoung Lee

alee@ewha.ac.kr

1 Department of Social Work, Kutztown University of Pennsylvania, 15200 Kutztown Rd, Kutztown, PA 19530, USA

2 Ewha Institute for Age Integration Research, Ewha Womans University, 52, Ewhayeodae-gil, Seodaemun-gu, Seoul 03760, Korea
Abuse issued new telehealth guidelines permitting the use of smart phones and other electronic devices, expanded the practitioner types that can utilize telehealth beyond clinically licensed staff to include behavioral health services that may be provided by unlicensed staff as long as their work is within their scope of practice, expanded the types of billable services that were previously only billable for in-person visits, eliminated the requirement that a certain number of sessions be in-person, and temporarily suspended the amount of services providers can bill under telehealth (Houser, 2020). Patel et al. (2021) reported that during the pandemic, among a national sample of 16.7 million individuals, 30.1 percent of all outpatient visits were provided via telemedicine, and the weekly number of telehealth visits increased twenty-threefold compared with the pre-pandemic period. According to them, 53\% of visits for depression were provided via telemedicine during the pandemic period.

Telehealth has become an integral part of mental health care delivery amid the pandemic replacing in-person, faceto-face interaction, and, in many cases, preventing the 
disruption of service provision. Even before the pandemic, telehealth offered a long held promise to mitigate and remediate urban-rural healthcare disparities perpetuated by rural residents' barriers to accessing mental health care services due to shortage of mental health professionals, lack of transportation, and long travel distances (Harrison \& Lee, 2006; Myers, 2019; Swinton et al., 2009; Weaver \& Himle, 2017). Scholars have pointed out the potential of telepsychiatry in collaboration with mental health professionals to increase mental health services to rural areas (Saeed \& Pastis, 2018).

Although telehealth is suggested to increase access to mental health services for underserved rural populations, there is a general consensus in the literature that telehealth does not benefit everyone equally, and underserved, rural populations are at a particular disadvantage (Park et al., 2018). A 2011 national survey of America's emerging online experience conducted by the U.S. Department of Commerce (2013) revealed that the majority of telemedicine users live in urban areas. According to the study, urban populations were twice as likely to engage in online healthcare activities when compared with rural telemedicine users. These trends are echoed in a recent study revealing that respondents living in rural areas were less likely to use telehealth than their urban counterparts (Jaffe et al., 2020). Many scholars concluded that the key reasons for low utilization rates of telemedicine among rural populations can mainly be explained by the shortage of mental health specialists offering this service, the absence of coverage and reimbursement, licensure issues, broadband access and adequacy, access to appropriate technology, and privacy and security concerns (Fortney et al., 2015; Goins et al., 2001; Lambert et al., 2016; Mehrotra et al., 2017; Myers, 2019; Park et al., 2018; Patient Engagement HIT, 2020).

Both youth and elderly are vulnerable populations with specific mental health needs such as loneliness, depression, and anxiety (Qualter et al., 2015), who are reported to have less access to health care among rural residents (Reiss, 2013). Youth from economically impoverished areas are at the most risk for ongoing mental health problems (Reiss, 2013), yet they often receive ineffective psychological treatment (Garland et al., 2013) due to inadequate and subpar services, as well as inadequate access to resources, such as transportation (Kodet et al., 2019). Barriers for the rural older adult population include service access issues related to rural residency, inability to recognize need for help, reluctance to ask for help, inability to leave their home, cost of treatment, diagnoses, and ability to participate in treatment, among others (Bischoff et al., 2014; Levesque et al., 2013).

Additionally, concerns about privacy, security, access to necessary technology, and a lack of familiarity engaging in treatment using internet platforms are barriers for youth and senior citizens alike (Guo et al., 2020; Kavandi \& Jaana, 2020; Reed et al., 2020). Two particularly concerning contextual issues for clinicians include the lack of adequate or available technology and working with high-risk populations that lack a confidential environment for treatment such as children and families experiencing domestic violence or abuse (Racine et al., 2020). Children with impulse control or self-regulatory problems may find sitting in front of a computer screen a challenge as well (Madigan, 2021). The ability of older adults to access and use technology is a widely discussed barrier to telehealth utilization. Feelings of apprehension in its use, lack of interest, and difficulty in learning how to use technology are reported as reasons for the limited use of technology among the elderly (Martson et al., 2019).

Acknowledging both the existing challenges and the opportunities of using telemental health services in rural areas, this study aimed to examine the experiences of mental health service and public insurance providers providing telemental health services amid the COVID-19 pandemic with special focus on rural youth and the elderly.

\section{Methodology}

The data was collected by focus group interviews with mental health service providers and representatives from Medicaid managed care organizations (MCOs) in Pennsylvania. MCOs and service providers were included in this study because these two groups must work together to come to agreement about how services will be provided in the community to ensure mental health and substance abuse services are accessible in rural Pennsylvania. This process requires cooperation and mutual agreement between service providers who provide the care and insurance companies who pay for the care. In order to understand barriers to accessing services, it is critical to understand the needs of MCO's, operating on a macro level, and service providers operating on the micro and mezzo level. To better understand how MCO and service provider needs manifest, we presented each group with a similar set of questions about service systems.

This qualitative data collection was part of a larger statewide, mixed-method study exploring access to and delivery of mental health services in rural Pennsylvania, with a specific focus on youth 18 and under and senior citizens 65 and older.

\section{Participants}

A purposive sampling strategy was employed to identify and recruit rural mental health service providers and insurance agency representatives for this study. To define rurality, we followed the Center for Rural Pennsylvania (CRP)'s rural/ urban definition. The CRP defines rural/urban based on the population density. The State of Pennsylvania's overall 
population density based on the 2010 Census is 284 persons per square mile. CRP defines a rural county when a county has less than 284 persons per square mile. Based on the definition, there are 48 rural counties and 19 urban counties in Pennsylvania (Center for Rural Pennsylvania, 2021). With this definition of rural county, we complied the list of mental health service providers in rural counties through the Department of Human Services of Pennsylvania website, Office of Mental Health and Substance Abuse Services on their "Human Services Provider Directory." Service provider focus group participants represented a wide variety of roles and responsibilities from agencies serving youth and elderly populations. The diverse experiences of focus group participants ranged from CEO/CFO level to clinical directors and direct care providers from public and private provider groups such as county mental health, area agency on aging, county crisis intervention, county case management, school systems, multicounty private mental health agencies, hospital case managers, counselors, and private practice practitioners.

Health insurance providers were identified using the National Committee for Quality Assurance (NCQA) report card for health plans. Care managers and clinical supervisors were invited to join the focus groups. Although this study attempted to recruit public and private insurers, repeated requests made to private insurers did not result in their participation due to expressed legal concerns. Medicare MCOs were also invited but did not participate. In the end, only Medicaid managed care insurance companies from the Pennsylvania Health Choices program are represented in this study. Although some of the Medicaid MCO representatives had knowledge and/or experience working with Medicare populations, failure in recruiting Medicare MCO representatives is the limitation of the study.

\section{Data Collection}

This study was approved by the Institutional Review Board at the University. Twenty-five focus groups with 119 service providers and 6 focus groups with 28 representatives comprising all of the Medicaid managed care organizations in the Health Choices program were conducted from June until October 2020. The focus groups were conducted online in real time using Zoom teleconferencing technology and lasted approximately $1-1.5 \mathrm{~h}$. Twenty randomly selected mental health service provider representatives who participated in the focus groups were randomly selected to receive a $\$ 25$ gift card as an incentive for participation. Each session was audio-recorded and auto-transcribed by Zoom. Each transcript was then reviewed and cleaned to prepare it for analysis in NVivo 12 qualitative analysis software. The codebook was developed on an ongoing basis until all the focus groups were completed. Saturation was achieved at the completion of all focus groups confirming repetitive emerging themes and conclusions and assuming that further data collection would yield similar results.

\section{Data Analysis}

This study adopted the analytic techniques of grounded theory wherein both inductive and deductive approaches to data analysis were used. The coding process started from a set of a-priori codes that were developed by the research team after each focus group with several codes emerging during the actual coding process. Each focus group interaction was considered as a unit of meaning and coded accordingly assigning as many codes as was deemed relevant by each independent coder.

One research assistant coded all the transcripts, and seven transcripts (23\% of the total documents) were selected for intercoder assessment. This intercoder sample is in line with the literature suggesting that samples of $10-25 \%$ of data are acceptable for conducting intercoder process (Campbell et al., 2013). After a norming session, the intercoder agreement was calculated in NVivo software based on paragraph by paragraph coding of transcripts. The coding comparison produced $96.7 \%$ agreement over seven transcripts. The literature suggests that a minimum of $80 \%$ agreement is required (Wilson-Lopez et al., 2019) or a range of 74-94\% agreement is necessary to be considered acceptable (Campbell et al., 2013). The overall Cohen's Kappa score was 0.588, reflecting moderate agreement (Burla et al., 2008). This, in part, can be explained by sensitivities related to occasionally selecting different units of meaning while coding as well as professional backgrounds of the coders (clinical/non-clinical background).

\section{Findings}

Two core themes and seven sub-themes emerged with regard to the use of telehealth and heavy reliance on technology for mental health care provision amid the COVID-19 pandemic: (1) silver linings during the pandemic and (2) some roadblocks to success. Although the themes emerged from participants serving youth and older adults, older adults experienced more barriers to using telehealth so providers had fewer insights to silver linings related to serving older adults during COVID-19.

\section{Silver Linings During the Pandemic}

The focus group participants widely shared perceived promising effects of telehealth in the forms of: (1) service continuation during the pandemic, (2) improved parental involvement and responsiveness, (3) easing the transportation challenges, and (4) decrease in no-show rates. 


\section{Service Continuation During the Pandemic}

Most of the focus group participants saw some silver linings amid the rapid changes and need for instant adaptation in service delivery associated with the sudden onset of the COVID-19 pandemic. Technology has allowed some service providers to adapt quickly to the changing times, as one service provider reported:

[front desk staff] really worked hard to contact every single person on our schedule, give them instructions. We also use a text; we use a texting platform. So, we were able to text them the, you know, the sites to go to and the instructions and so we use different you know phone and texting media to get that word out.

An insurance provider commented on the perceived utility and effectiveness of telehealth for the youth population receiving mobile services:

A lot of the children received mobile services. So they're in home or in the community, which is really beneficial, especially in rural areas. So the expansion of telehealth allowed the providers to be able to stay in touch and stay in contact with those families ... but so telehealth was important to be able to continue to try to maintain contact....

As such, agencies adapted quickly to mobile/virtual service provision to continue mental health service delivery during the covid-19 crisis and service providers acknowledged it was a critical solution to continue services to the clients.

\section{Improved Parental Involvement and Responsiveness}

The second silver lining during the pandemic that the participants mentioned was that the convenience of telehealth resulted in improved parental engagement and responsiveness. An insurance provider shared:

I would say that for my team working with familybased providers... in the perfect world, they're meeting with kids and their families in their home or community setting and then... all of a sudden the family-based teams were using Telehealth options. And we've been hearing for the last six months about some emerging best practices around that, silver linings for sure that, you know, family members who previously would have done everything they could do to avoid meeting with the family-based therapists are more engaged when it's a screen and just somehow that mechanism just worked better for that person to be involved in the treatment...

Similarly, a service provider further explained that busy schedules of parents and time to travel to appointments served as impediments to service use pre-pandemic, and the use of telehealth helped increase convenience and hence parental responsiveness:

... oftentimes with COVID the families are more responsive because it's more convenient. They don't have to take time out of their schedule to schedule an appointment and go travel to an office and meet with a team or whatnot, you know, they find it more convenient to just do a zoom session. So it's actually been a bit better.

\section{Easing the Transportation Challenges}

Many service providers shared that in addition to improved parental involvement, they observed families appreciate telehealth as a convenient solution to the perennial transportation problem and long travel distance and time to get to appointments in rural areas. Participants discussed the impact of telehealth on easing the transportation barrier for many clients by enabling them to access services virtually. Telehealth was also reported as a means to save travel time to attend appointments. This is summarized well in the words of this insurance provider:

Some families have been more willing, and things could be scheduled when maybe more of the family was available and could interact via the telehealth the therapist also doesn't have that travel time between the homes. So, there's, you know, a little bit of a little bit more leeway in, you know how many sessions, they might be able to have or that kind of thing. And obviously, face to face is still, you know, ideally the ultimate goal, but there's been some definite benefits. I think telehealth has also addressed some access issues and of course this has been because of COVID but families who may not have been able to get to transportation get to a psychologist for an evaluation or to a psychiatrist for an evaluation... they've been able to do it via telehealth.

\section{Decreased Number of No-Show Rates}

According to service and insurance providers, all the abovementioned outcomes lead to noticeable drops in cancellations and missed appointments. As one service provider explained:

Our experience was through COVID particularly when things were closed down. Everybody was closed down. We were at full speed ahead and almost had a sense that because folks could not be out and about, they were much more likely to have no reason to cancel and 
were seeking out. Even the telehealth appointments so that we had a very low rate of cancellation or no show.

Another provider pointed out that the decrease in cancellations and no-show rates as an outcome of relying on telehealth has helped providers stay in business and sustain service delivery:

It has also helped providers who are operating on a shoestring they're not really making a lot of money working in the mental health field without patient services so that they're no-show rates have greatly decreased, which is enabling them to bill and be more sustaining of the services they have.

Overall, telehealth technology has provided momentous solutions to maintain mental health service delivery to rural areas during the sudden COVID-19 crisis with the State's stay-at-home order. Further, it was reported to enhance convenience for service users and remove service access obstacles such as transportation issues, long travel distance and time, and resulted in a decrease of no-show to mental health sessions. It is important to emphasize that these silver linings were reported to mostly benefit families with youth, not the older adult population.

\section{Some Roadblocks to Success}

While telehealth was generally seen as a necessary service delivery alternative amid the pandemic and a helpful solution for some of the service access issues, focus group participants also discussed associated challenges and provided critiques. Participants reported challenges associated with the use of different technological platforms that may prevent interaction, engagement, and effectiveness of service. Service providers also reported that service users not owning the latest technology and having pre-paid phone plans interfered with service continuation and quality. Sub-themes in this section are (1) Not for every youth! (2) Technology challenges among the older adults, and (3) "Dead zones" without internet and cellphone reception.

\section{Not for Every Youth!}

There were varying levels of telehealth acceptability based on the level of functioning of the children. When discussing the application of telehealth in work with children with disabilities, service and insurance providers questioned its utility. In general, telehealth was seen as creating barriers to service delivery for children who have disabilities as it is nearly impossible to engage them and replicate effective interaction techniques used with this population when teaching social skills. A service provider explained that:
Working on social skills with children with autism, for example, is next to impossible via Zoom depending on where they're at on the spectrum. Some of your higher functioning kids might be all about it and super engaged with you and it's a great tool. And the next kid who's nonverbal and further down in functioning and the spectrum doesn't engage at all. And therefore, you know, that becomes an unusable delivery method for them, you know, we've seen the entire gamut.

Service and insurance providers alike reported challenges associated with the use of different technological platforms that may prevent interaction, engagement, and effectiveness of service. An insurance provider reported that

There are some kids who are more reachable when you're interacting with them through zoom or FaceTime, or any of those other things. And then certainly some downsides, you know, for kids that have an intellectual disability or on the autism spectrum. Sometimes it's much harder to engage them. So definitely silver linings but certainly not for everybody.

Similar to how use of technology for service provision might not work for and benefit equally all populations, its utility and effectiveness were also reported to not be equally advantageous when applied for different therapeutic interventions. For example, it was pointed out that providing therapeutic staff support (TSS) or partial hospitalization programs were difficult with telehealth. An insurance provider shared the following:

Now not every family was open to doing specific services like well TSS is probably the best example of a service that was sort of hard to figure out how that would look via tele health, simply because it's usually much more hands on you know there's a lot of prompting redirection in person. So for the providers that weren't going into the home, a lot of times, families, would decline the TSS to continue at that point. (...) The same was true for the Child and Adolescent partials that you know clearly. If a kiddo needed to be in partial they typically needed to be there for the full day and participate and trying to engage you know, a kiddo via tele health or video for what partial typically provides was difficult, especially to just engage them for the time that they even needed to bill for services and you know the benefit wasn't necessarily there that in person partial hospitalization programs. (...)

These challenges associated with using technology in mental health services were also evident with the older population. 


\section{Technology Challenges Among the Older Adults}

The use of technology was reported to be fairly new for the elderly population, a factor that can cause significant barriers in service delivery. Elderly clients not owning the most recent and appropriate technology was seen as a particular impediment along with low comfort levels with the new, unfamiliar method of care and distrust of technology. One insurance provider shared:

Well piggybacking on the comments... when you know they may not have the right technology or know how to use it properly to have these types of calls if it needs to be a face to face, not just a telephone call. So that is a barrier for a number of people. Or, you know, they just don't feel comfortable doing that. That's not how, you know, the older population if they like that face to face, and they don't feel that they're getting the same level of service, you know, not having that of an intimate environment... on the phone.

As stated above, there is a cultural normality and generational comfort of face-to-face services for the older population in which services provided through technology may cause them to feel uncomfortable or unwilling to receive services. A service provider similarly reported:

And again, it was an adjustment for our elderly population because they're so used to having everything on paper. So that was a big change and a big challenge of, you know, calling a managed care organization and you have five things to choose from. You know, press this if you're a participant, press this if you're a provider. They just want to talk to somebody and say my agent show up.

Due to the pandemic-related service delivery changes, the elderly were reported to experience barriers when technology is used for service provision. Both insurance providers and service providers expressed that because of these factors, the continuity of serving the elderly population was challenging during the COVID-19 crisis. Overall, reliance on telehealth as a primary mode of service delivery appears to be far from optimal when working with elderly and children with disabilities who need mental health services.

\section{"Dead Zones" Without Internet and Cellphone Reception}

Some of our participants referred to rural areas where cellphone reception is limited and internet access may not be available as "dead zones." These conditions were reported to create significant limitations for both service access and delivery. One service provider reported that these issues were clearly exposed amid the pandemic:
One of the barriers we have up here is the lack of infrastructure, the technology is there-the zoom and telehealth-however, we don't have the data service, the internet service. We have a great number of dead zones in [name of the county] and [name of the county] where there is no access and availability and the lack of infrastructure... the pandemic has really put a spotlight on that, that we don't have cell coverage. We don't have high speed access even here in our office in town. If too many people use zoom it can crash.

Similarly, an insurance provider stated:

And then, you know, the phone services in a lot of places. If you drive through a lot of rural Pennsylvania, you can't even, you know, make the cell phone call. So even in, you know, in this time of COVID-19 we've been... doing some Telehealth services. That poses a challenge when there's no internet service, there's no cell service. So, yeah, that can be very challenging.

Rural "dead zones" create serious barriers to access for service recipients and to service delivery for service and insurance providers. The challenges of "dead zones" result in the interruption of communication between insurers, service providers, and consumers which directly impacts the delivery of care.

Overall, based on the experiences and perspectives of service providers and public insurance representatives, telehealth was reported as a promising modality of service delivery, albeit not for all populations. They suggested considering hybrid models of service delivery and finding a way to utilize telehealth as an alternative, billable modality of service delivery beyond the pandemic.

.... lot of it's been sort of on an emergency basis because of the need, I do think that there's been discussion that moving forward. If we're ever beyond COVID that there you know may ideally be some consideration to continue some telehealth services because of some of the positives. But you know, I think that that would be as far as quality measures and things that would have to be something that would be set moving forward. I'm not so sure that it's being monitored at this point, just because we're trying to get services in place, somehow, because of COVID.

\section{Discussion and Implications}

The COVID-19 pandemic appears to have created an unprecedented scope for testing the utility and effectiveness of telehealth as a remote modality of mental health service provision. This study reported the findings from a state-wide, 
exploratory study of mental health professionals focusing on their practice experiences and perspectives using telehealth amid the COVID-19 pandemic. Our study participants perceived telehealth as both: silver linings during the pandemic and some roadblocks to success.

As reported in previous studies (Reay et al., 2020), our research similarly shows that telehealth appears to be a promising, alternative mode of service delivery that cuts across several service access barriers in rural areas, including lack of public transportation, long travel distances, and limited availability of mental health professionals physically located in rural communities. We also found that based on the practice experiences of service providers, telehealth appeared to work well for youth and was reported to improve parental responsiveness and engagement in mental health care due to its convenience. Many mental health service providers also reported a decrease in appointment cancellations and no-show rates as one of the unintended consequences related to telehealth use. These findings appear to be contributing to the existing knowledge on the promising, positive effects of telehealth and should be explored in future research. However, it stands to reason that the impact of improved participation could be unique to the COVID-19 circumstances due to stay-at-home orders and parental flexibility related to working from home-a phenomenon that might not hold once parents return back to work in person.

Notably, telehealth is not a panacea as it does not work equally well for all mental health populations and all therapeutic modalities and is not a reliable option in remote, "dead zones" of rural areas. Specifically, telehealth was perceived as a substandard modality for the elderly due to the limited access to and proficiency using technology-a limitation that has been discussed extensively in the existing literature (e.g., Iancu \& Iancu, 2020; Martson, et al., 2019). Contrary to these findings and general popular belief regarding the challenges of older adults in technology use, Anderson and Perrin (2017) have documented an increase in "tech adoption" among elderly Americans. This fact may serve as an impetus for more widespread outreach, education, and infrastructure support to facilitate access to mental health services among rural older individuals. Strategies to address the underserved rural older adult population have been under debate for a significant period of time, albeit with limited breakthroughs and minimal empirical testing as compared to the youth population using telehealth services (e.g. Anderson et al., 2017; Comer et al., 2017; Myers et al., 2015; Tse et al., 2015). In rural areas, where scarcity of quality and age-appropriate care as well as transportation and lengthy travel distance issues are especially rife, telemedicine solutions may offer a way forward in addressing diverse needs of the elderly population (Goins et al., 2001).

Telehealth was also seen as a suboptimal mode of service delivery for children with intellectual disabilities or those with autism spectrum diagnoses and for certain therapeutic modalities, such as TSS and partial hospitalization program and social skill building interventions. The latter findings are scarcely covered in the existing literature and need to be explored in-depth in future research focusing on telehealth application for diverse mental health populations and using different therapeutic interventions. Service providers in our study also reported that clients with older technology and having pre-paid phone plans interfered with remote service continuation and quality of care. Lastly, our participants provided ample discussion on the need for advanced infrastructure to improve access to internet and cell phone reception in "dead zone" rural areas. The concerns related to reliable infrastructure, access to technology, and high-speed internet have been previously discussed as significant barriers to the adoption and implementation of telehealth on a large scale (e.g. Goins et al., 2001; Myers, 2019).

Service providers and insurers in our study advocated that policy makers keep telehealth as a billable option for mental health service provision beyond the COVID-19 emergency, particularly for populations to whom it appears to be beneficial. It is incumbent upon state legislators, insurance companies, and service providers to find some way to utilize telehealth in ways that address some of the transportation, scheduling, and basic need insecurities preventing families from engaging in mental health services. Policy makers and local officials should consider investing in infrastructure development to improve cell phone reception and internet access in rural areas. We recommend that legislators consider passing telemedicine parity legislation allowing mental health and substance abuse treatment providers to be reimbursed for telehealth services similarly to face-to-face treatment beyond the pandemic and incentivizing innovative solutions for telehealth services when serving adults $65+$ and youth $\leq 18$.

Mehrotra et al. (2017) found that telemental health was used more in states with a telemedicine parity law and protelemental health regulations. This finding supports our policy recommendation to keep telehealth as a reimbursable service beyond COVID-19 pandemic and to facilitate legislative action to pass the state telemedicine parity laws. Surprisingly, a study conducted by Park and colleagues (2018) found no association between less restrictive state telehealth policies and increased telehealth usage among any populations. This finding suggests that state efforts alone to ease policies and remove barriers to using telehealth might not be sufficient for increasing telehealth use; financial incentives for providers to adopt telehealth and removing copayments for virtual visits for consumers to increase utilization may be needed (Park et al., 2018).

The current study has several notable limitations that offer direction for future studies. Data of the current study were derived from a limited sample population obtained 
from the state of Pennsylvania. In addition, insurers in this study only represent medicaid managed care organizations which may view telehealth differently than Medicare managed care organizations and private insurance companies. Future studies ought to attempt to focus efforts to engage diverse insurance companies. Focus group data for this study is also limited to the experiences of service providers but not the people receiving mental health services. While mental service providers feel telehealth services potentially increase access to mental health and substance abuse treatment for people living in rural communities, this study lacks data about how these services are received by service users. Future studies ought to include attitudes of recipients of telehealth services to determine whether the benefits of telehealth identified by mental health service providers and managed care organizations align with the lived experience of people receiving those services.

\section{Conclusion}

Despite these limitations, this study captured the experiences of mental health professionals who were engaged in telemental health during the pandemic period in various rural counties in Pennsylvania. Participants represented direct care service providers and agency administrators of organizations serving youth and older adults. This study provides a timely description of opportunities and challenges facing service providers using telemental health in rural areas. We have identified some silver linings associated with telehealth use amid the pandemic, but caution against treating telehealth as a panacea. Policy and practice recommendations include incentives for proactive telehealth uptake, telehealth parity laws and reimbursement policies, and incentivizing innovative use of technology for specific populations and therapeutic modalities. Continuous policy support and organizational efforts to provide customized telemental health are called for to remediate rural disparities in access to mental health services beyond the pandemic period.

Funding This project was made possible, in part, by a grant from the Center for Rural Pennsylvania, a legislative agency of the Pennsylvania General Assembly under Grant 2020-6; and the National Research Foundation of Korea (NRF) Grant funded by the Korea government (No. 2020S1A5C2A03092919).

\section{Declarations}

Conflict of interest The authors have no relevant financial or non-financial interests to disclose.

Ethical Approval Approval was obtained from the ethics committee of the Kutztown University of Pennsylvania. The procedures used in this study adhere to the tenets of the Declaration of Helsinki.
Consent to Participate Informed consent was obtained from all individual participants included in the study.

\section{References}

Anderson, K., Byrne, C., Crosby, R., \& LeGrange, D. (2017). Utilizing telehealth to deliver family-based treatment for adolescent anorexia nervosa. International Journal of Eating Disorders, 50(100), 1235-1238.

Anderson, M. \& Perrin, A. (2017). Tech adoption climbs among older adults. Retrieved from https://www.pewresearch.org/internet/ 2017/05/17/tech-adoption-climbs-among-older-adults/. Accessed 28 Mar 2021.

Bischoff, R. J., Reisbig, A. M., Springer, P. R., Schultz, S., Robinson, W. D., \& Olson, M. (2014). Succeeding in rural mental health practice: Being sensitive to culture by fitting in and collaborating. Contemporary Family Therapy, 36(1), 1-16.

Burla, L., Knierim, B., Barth, J., Liewald, K., Duetz, M., \& Abel, T. (2008). From text to codings: Intercoder reliability assessment in qualitative content analysis. Nursing Research, 57(2), 112-118.

Campbell, J., Quincy, C., Osserman, J., \& Pedersen, O. (2013). Coding in-depth semistructured interviews: Problems of unitization and intercoder reliability and agreement. Sociological Methods and Research, 42(3), 294-320.

Centers for Medicare \& Medicaid Services. (2020). President Trump expands telehealth benefits for medicare beneficiaries during COVID-19 outbreak. Newsroom. Retrived March 17, 2020, from https://www.cms.gov/newsroom/pressreleases/president-trumpexpands-telehealth-benefits-medicare-beneficiariesduring-covid19-outbreak. Accessed 01 Mar 2021.

Center for Rural Pennsylvania. (2021). Rural urban definitions. Retrieved from https://www.rural.palegislature.us/demographi cs_rural_urban.html. Accessed 13 July 2021.

Comer, J., Furr, J., Kerns, C., Miguel, E., Coxe, S., Elkins, R., Carpenter, A., Cornacchio, D., Cooper-Vince, C., DeSerisy, M., Chou, T., Sanchez, A., Khanna, M., Franklin, M., Garcia, A., \& Freeman, J. (2017). Internet-delivered, family-based treatment for early-onset OCD: A pilot randomized trial. Journal of Consulting and Clinical Psychology, 85(2), 178.

Fortney, J. C., Pyne, J. M., Turner, E. E., Farris, K. M., Normoyle, T. M., Avery, M. D., Hilty, D. M., \& Unützer, J. (2015). Telepsychiatry integration of mental health services into rural primary care settings. International Review of Psychiatry, 27(6), 525-539. https://doi.org/10.3109/09540261.2015.1085838

Garland, E. L., Pettus-Davis, C., \& Howard, M. O. (2013). Self-medication among traumatized youth: Structural equation modeling of pathways between trauma history, substance misuse, and psychological distress. Journal of Behavioral Medicine, 36(2), 175-185.

Goins, R. T., Kategile, U., \& Dudley, K. C. (2002). Telemedicine, rural elderly, and policy issues. Journal of Aging \& Social Policy, 13(4), 53-71.

Guo, X., Lin, H., Wu, Y., \& Peng, M. (2020). A new data clustering strategy for enhancing mutual privacy in healthcare IoT systems. Future Generation Computer Systems, 113, 407-417. https://doi. org/10.1016/j.future.2020.07.023

Harrison, J. P., \& Lee, A. (2006). The role of e-health in the changing health care environment. Nursing Economics, 24(6), 283-288.

Houser, K. (2020). Telehealth guidelines related to COVID-19 (updated). Pennsylvania Press Room. Retrieved from https://www. dhs.pa.gov/providers/Providers/Documents/Coronavirus\%202020/ OMHSAS\%20COVID-19\%20Telehealth\%20Expansion-\%20Fin al\%203.15.20.pdf. Accessed 15 July 2021. 
Iancu, I., \& Iancu, B. (2020). Designing mobile technology for elderly. A theoretical overview. Technological Forecasting and Social Change, 155, 117. https://doi.org/10.1016/j.techfore.2020.119977

Jaffe, D. H., Lee, L., Huynh, S., \& Haskell, T. P. (2020). Health inequalities in the use of telehealth in the United States in the lens of COVID-19. Population Health Management, 23(5), 368-377.

Kavandi, H., \& Jaana, M. (2020). Factors that affect health information technology adoption by seniors: A systematic review. Health \& Social Care in the Community, 28(6), 1827-1842.

Kodet, J., Reese, R. J., Duncan, B. L., \& Bohanske, R. T. (2019). Psychotherapy for depressed youth in poverty: Benchmarking outcomes in a public behavioral health setting. Psychotherapy, $56(2), 254$.

Lambert, D., Gale, J., Hartley, D., Croll, Z., \& Hansen, A. (2016). Understanding the business case for telemental health in rural communities. The Journal of Behavioral Health Services \& Research, 43(3), 366-379.

Levesque, J. F., Harris, M. F., \& Russell, G. (2013). Patient-centered access to health care: Conceptualizing access at the interface of health systems and populations. International Journal for Equity in Health, 12, 18. https://doi.org/10.1186/1475-9276-12-18

Madigan, S., Racine, N., Cooke, J. E., \& Korczak, D. J. (2021). COVID-19 and telemental health: Benefits, challenges, and future directions. Canadian Psychology/Psychologie Canadienne, 62(1), 5-11.

Marston, H. R., Genoe, R., Freeman, S., Kulczycki, C., \& Musselwhite, C. (2019). Older adults' perceptions of ICT: Main findings from the technology in later life (TILL) study. Healthcare, 7(3), 86. https://doi.org/10.3390/healthcare7030086

Mehrotra, A., Huskamp, H. A., Souza, J., Uscher-Pines, L., Rose, S., Landon, B. E., ... \& Busch, A. B. (2017). Rapid growth in mental health telemedicine use among rural Medicare beneficiaries, wide variation across states. Health Affairs, 36(5), 909-917.

Myers, C. R. (2019). Using telehealth to remediate rural mental health and healthcare disparities. Issues in Mental Health Nursing, 40(3), 233-239.

Myers, K., Vander Stoep, A., Zhou, C., McCarty, C., \& Katon, W. (2015). Effectiveness of a telehealth service delivery model for treating attention-deficit/hyperactivity disorder: A communitybased random controlled trial. Journal of the American Academy of Child and Adolescent Psychiatry, 54(4), 263-274.

Park, J., Erikson, C., Han, X., \& Iyer, P. (2018). Are state telehealth policies associated with the use of telehealth services among underserved populations? Health Affairs, 37(12), 2060-2068.

Patel, S. Y., Mehrotra, A., Huskamp, H. A., Uscher-Pines, L., Ganguli, I., \& Barnett, M. L. (2021). Variation in telemedicine use and outpatient care during the COVID-19 pandemic in the United States: Study examines variation in total US outpatient visits and telemedicine use across patient demographics, specialties, and conditions during the COVID-19 pandemic. Health Affairs, 40(2), 349-358.

Patient Engagement HIT. (2020). Reducing care disparities for rural populations with telehealth. Retrieved from https://patienteng agementhit.com/resources/webcasts/panel-reducing-care-dispa rities-for-rural-populations-withtelehealth. Accessed 12 Feb 2021.
Qualter, P., Vanhalst, J., Harris, R., Van Roekel, E., Lodder, G., Bangee, M., \& MaesVerhagen, M. M. (2015). Loneliness across the life span. Perspectives on Psychological Science, 10(2), 250-264.

Racine, N., Birken, C., \& Madigan, S. (2020). Supporting the mental health of parents and children during and after coronovirus. Journal of Developmental \& Behavioral Pediatrics, 41(7), 508-510.

Reay, R., Looi, J., \& Keightley, P. (2020). Telehealth mental health services during COVID-19: Summary of evidence and clinical practice. Australian Psychiatry, 28(5), 514-516.

Reed, N. S., Ferrante, L. E., \& Oh, E. S. (2020). Addressing hearing loss to improve communication during COVID-19 pandemic. Journal of the American Geriatrics Society, 68, 1924-1926.

Reiss, F. (2013). Socioeconomic inequalities and mental health problems in children and adolescents: A systematic review. Social Science \& Medicine, 90, 24-31.

Saeed, S. A., \& Pastis, I. (2018). Using telehealth to enhance access to evidence-based care. Psychiatric Times, 35(6), 9-22.

Swinton, J. J., Robinson, W. D., \& Bischoff, R. J. (2009). Telehealth and rural depression: Physician and patient perspectives. Families, Systems, and Health, 27(2), 172-182.

The Centers for Medicare and Medicaid Services. (2020). COVID-19 emergency declaration blanket Waivers for health care providers. Retrieved from https://www.cms.gov/files/document/summarycovid-19-emergency-declaration-waivers.pdf. Accessed 14 July 2021.

Tse, Y., McCarty, C., Vander Stoep, A., \& Myers, K. (2015). Teletherapy delivery of caregiver behavior training for children with attention-deficit hyperactivity disorder. Telemedicine and e-Health, 21, 451-458.

U.S. Department of Commerce. (2013). Exploring the digital nation: America's emerging online experience. Retrieved from https:// www.ntia.doc.gov/files/ntia/publications/exploring_the_digital_ nation_-_americas_emerging_online_experience.pdf accessed March 2, 2021.

Weaver, A., \& Himle, J. A. (2017). Cognitive-behavioral therapy for depression and anxiety disorders in rural settings: A review of the literature. Journal of Rural Mental Health, 41(3), 189-221.

Wilson-Lopez, A., Angela Minichiello, P. E., \& Green, T. (2019). An inquiry into the use of intercoder reliability measures in qualitative re-search. In ASEE Annual Conference proceedings. ASEE. Retrieved from https://par.nsf.gov/servlets/purl/10089476. Accessed 25 Apr 2021.

Wolf, T (2020). Proclamation of disaster. Newsroom. Retrieved from https://www.governor.pa.gov/newsroom/gov-wolf-signs-covid-19disaster-declaration-to-provide-increased-support-for-state-respo nse/. Accessed 13 July 2021.

Publisher's Note Springer Nature remains neutral with regard to jurisdictional claims in published maps and institutional affiliations. 